\title{
The New Combination of Semi-Analytical Iterative Method and Elzaki Transform for Solving Some Korteweg-de Vries Equations
}

\author{
Areej Salah Mohammed \\ E-mail: areej.s.m@ihcoedu.uobaghdad.edu.iq
}

\begin{abstract}
:
The purpose of this research applied a method that includes the combination of an iterative method (Temimi and Ansari method (TAM)) and a new transform called the Elzaki transform (ET )for some Korteweg-de Vries (Kdv) equations. The TAM was present as a basic tool for solving Kdv equations with Elzaki transformation in the associated nonlinear equations because the Elzaki transformation cannot deal with nonlinear terms. It has been proven that the use of this method can be more reliable, accurate and fast if it has been using analytical methods alone.
\end{abstract}

KEYWORDS: Elzaki Transformation, Semi-Analytical Iterative Method, Korteweg-De Vries Equations, Approximate Analytical Solutions, Nonlinear Equations.

\section{Introduction}

Many phenomena in physics, chemistry, biology, and mathematical engineering are represented by partial differential equations. For example, the dispersion of the reaction chemicals, matters related to population growth, fluid flow and heat diffusion are characterized by partial differential (PD) equations. On the other hand, modeling of physical phenomena emerged as partial non-linear differential equations an important tool in a large category and is widely used in various fields of natural nonlinear sciences, while the behavior and characteristics of non-linear PD equations are determined by the exact solution which is difficult to find in most, many researchers are interested in studying and solving these equations because of their different applications. For example but not limited to, [1], [2] , [3], [4]. The KdV equation is a model for describing weak non-linear long waves in many branches of physics and engineering. Hence, many research works have been invested in studying KDV equations, such as the Adomian decomposition method [5], the Homotopy Perturbation method [6], Temimi and Ansari method [7]. There are many attempts to combine two methods of solution, iterative methods with transformations such as the Laplace transform and the Elzaki transform, among others, Laplace Adomian decomposition method [8], [9], Homotopy Perturbation Transform Method [10], [11], Aboodh decomposition method[12], Elzaki decomposition method [13], [14].

Over the last few years, Temimi and Ansari Method (TAM), with it, an analytical solution to many nonlinear problems is found [15]. In this paper, we presented an approximate analytical tool that was developed in recent years to solve the equation specified in this research. The aim is to achieve a method more accuracy and getting highspeed convergence.

\section{Elzaki Transformation}

ET proposed by Tarig Elzaki [16], ET and some of its essential properties are used to solve differential equations.

We need to mention the basic definitions of this transformation as follows, the Elzaki transformation of the function $\mathrm{k}(\mathrm{r}, \mathrm{t})$ is

$\varepsilon[k(r, t)]=v \int_{0}^{\infty} k(r, t) e^{-\frac{t}{v}} d t=\mathrm{K}(r, v), t \geq 0$

Using integration by part, then we obtain the ET of partial derivatives

$\mathcal{E}\left[\frac{\partial k(r, t)}{\partial t}\right]=\frac{\mathrm{K}(r, v)}{v}-v k(r, 0)$,

$\mathcal{E}\left[\frac{\partial k(r, t)}{\partial r}\right]=\frac{d}{d x}[\mathrm{~K}(r, v)]$

$\mathcal{E}\left[\frac{\partial^{2} k(r, t)}{\partial t^{2}}\right]=\frac{\mathrm{K}(r, v)}{v^{2}}-k(r, 0)-v \frac{\partial k(r, 0)}{\partial t}$,

$\mathcal{E}\left[\frac{\partial^{2} k(r, t)}{\partial r^{2}}\right]=\frac{d^{2}}{d r^{2}}[\mathrm{~K}(r, v)]$

$\mathcal{E}\left[\frac{\partial^{n} k(r, t)}{\partial t^{n}}\right]=\frac{\varepsilon[k(r, t)]}{v^{n}}-\sum_{i=0}^{n-1} v^{2-n+i} \frac{\partial^{i} k(r, 0)}{\partial t^{i}}$

$\mathcal{E}\left[\frac{\partial^{n} k(r, t)}{\partial r^{n}}\right]=\frac{d^{n}}{d r^{n}}[\mathrm{~K}(r, v)]$

\section{The basic idea of TAM}

To presented the main idea of TAM, let us consider the general equation

$$
L(w(r, t))+N(w(r, t))+s(r, t)=0
$$

with boundary conditions $\quad B\left(w, \frac{\partial w}{\partial t}\right)=0$,

where $\mathrm{y}$ is unknown function, $\mathrm{L}$ is the linear operator, $\mathrm{N}$ is the nonlinear operator and $\mathrm{h}$ is a known function. 
Assuming that $w_{0}(r, t)$ is a solution of equation (2) of the initial condition,

$L\left(w_{0}(r, t)\right)+s(r, t)=0$, with $\left(w_{0}, \frac{\partial w_{0}}{\partial t}\right)=0$.

To find the next iteration we resolve the following equation:

$L\left(w_{1}(r, t)\right)+N\left(w_{0}(r, t)\right)+s(r, t)=$ $0, B\left(w_{1}, \frac{\partial w_{1}}{\partial t}\right)=0$.

Thus, an iterative procedure can be effective solution of the following problem,

$$
\begin{aligned}
& L\left(w_{n+1}(r, t)\right)+N\left(w_{n}(r, t)\right)+s(r, t)= \\
& 0, B\left(w_{n+1}, \frac{\partial w_{n+1}}{\partial t}\right)=0 .
\end{aligned}
$$

Each of $w_{i}$ are solutions to equation (1) [15].

\section{Algorithm of the Method ET}

Consider the following nonlinear partial differential equation

$$
\frac{\partial^{m} k(r, t)}{\partial t^{m}}=N(k(r, t))+s(r, t), m=1,2,3, \ldots
$$

$$
\text { with initial condition }\left.\frac{\partial^{m-1} k(r, t)}{\partial t^{m-1}}\right|_{t=0}=f_{m-1}(r) \text {, }
$$

In the beginning we find the first iteration by the TAM

$$
L\left(k_{0}(r, t)\right)=s(r, t)
$$

$$
\text { Where } L=\frac{\partial^{m}}{\partial t^{m}}
$$

It's clear that the integral operator $L_{t}{ }^{-1}$ exist and it's regarded as definite integral

$L_{t . . t}{ }^{-1}=\int_{0}^{t} \ldots \int_{0}^{t}() d. t \ldots d t$

And this means:

$$
k_{0}(r, t)=L_{t \ldots t}{ }^{-1}(s(r, t))
$$

(8)

Then we use the ET which defined in equation (1) on both aspects of equation (4) with respect equation (6)

$\mathcal{E}\left[\frac{\partial^{m_{k}} k_{1}(r, t)}{\partial t^{m}}\right]=\mathcal{E}\left[N\left(k_{0}(r, t)\right)\right]+\mathcal{E}[s(r, t)]$

$\frac{\varepsilon\left[k_{1}(r, t)\right]}{v^{n}}-\sum_{i=0}^{n-1} v^{2-n+i} \frac{\partial^{i} k_{0}(r, 0)}{\partial t^{i}}=\mathcal{E}\left[N\left(k_{0}(r, t)\right)\right]+$ $\mathcal{E}[s(r, t)]$

$k_{1}(r, t)=\mathcal{E}^{-1}\left[v^{n} \sum_{i=0}^{n-1} v^{2-n+i} \frac{\partial^{i} k_{0}(r, 0)}{\partial t^{i}}\right]+$ $\mathcal{E}^{-1}\left[v^{n} \mathcal{E}\left[N\left(k_{0}(r, t)\right)\right]\right]+\mathcal{E}^{-1}\left[v^{n} \mathcal{E}[s(r, t)]\right]$

The iterative procedure can be an efficient solution to the next problem

$$
k_{n+1}(r, t)=\mathcal{E}^{-1}\left[v^{n} \sum_{i=0}^{n-1} v^{2-n+i} \frac{\partial^{i} k_{n}(r, 0)}{\partial t^{i}}\right]+
$$$$
\mathcal{E}^{-1}\left[v^{n} \mathcal{E}\left[N\left(k_{n}(r, t)\right)\right]\right]+\mathcal{E}^{-1}\left[v^{n} \mathcal{E}[s(r, t)]\right]
$$

\section{Numerical Results}

The effectiveness of the method is verified by finding the solutions of KDV equations in this section.

Example 1. Consider the following homogeneous KDV equation

$k_{t}=-6 k k_{r}-k_{r r r}$, with initial condition $k(r, 0)=r$,

The initial problem is

$L\left(k_{0}(r, t)\right)=0$,

Then, we get

$k_{0}(r, t)=r$,

And the first iteration will be:

$k_{1_{t}}=-6 k_{0} k_{0_{r}}-k_{0_{r r r}}$,

Applying ET to both aspects

$\varepsilon\left[k_{1 t}\right]=\varepsilon\left[-6 k_{0} k_{0_{r}}\right]-\varepsilon\left[k_{0_{r r r}}\right]$

Depending on the ET laws, we get:

$\frac{\mathrm{K}_{1}(r, v)}{v}-v k_{1}(r, 0)=\varepsilon\left[-6 k_{0} k_{0_{r}}\right]-\varepsilon\left[k_{0_{r r r}}\right]$,

Then we use the initial condition with simplification, and take the inverse of ET we get:

$k_{1}=\mathcal{E}^{-1}\left[v^{2} r\right]+\mathcal{E}^{-1}[v \mathcal{E}[-6 r]]$,

$k_{1}=(1-6 t) r$,

In the same way, we obtain

$k_{2}=\left(1-6 t+36 t^{2}-72 t^{3}\right) r$,

$k_{3}=\left(1-6 t+36 t^{2}-216 t^{3}+864 t^{4}-\cdots\right) r$,

The closed-form solution will be

$k=\frac{r}{1+6 t}$.

Example 2. Consider the following homogeneous fifth order KDV equation

$k_{t}=-k k_{r}-k k_{r r r}+k_{r r r r r}$,

With initial condition $k(r, 0)=e^{r}$,

The initial problem is

$L\left(k_{0}(r, t)\right)=0$,

Then, we get

$k_{0}(r, t)=e^{r}$,

And the first iteration will be:

$k_{1_{t}}=-k_{0} k_{0_{r}}-k_{0} k_{0_{r r r}}+k_{0_{r r r r}}$,

Applying ET to both aspects

$\varepsilon\left[k_{1 t}\right]=\varepsilon\left[-k_{0} k_{0_{r}}\right]-\varepsilon\left[k_{0} k_{0_{r r r}}\right]+\varepsilon\left[k_{0_{r r r r}}\right]$,

Depending on the ET laws, we get:

$\frac{\mathrm{K}_{1}(r, v)}{v}-v k_{1}(r, 0)=\varepsilon\left[-k_{0} k_{0 r}\right]-\varepsilon\left[k_{0} k_{0 r r r}\right]+\varepsilon\left[k_{0 r r r r}\right]$,

dependent to the initial condition with simplification and take the inverse of ET we get:

$k_{1}=\mathcal{E}^{-1}\left[v^{2} e^{r}\right]+\mathcal{E}^{-1}\left[v \mathcal{E}\left[e^{r}\right]\right]$,

$k_{1}=(1-t) e^{r}$,

In the same way we obtain

$k_{2}=\left(1-t+\frac{t^{2}}{2 !}\right) e^{r}$,

$k_{2}=\left(1-t+\frac{t^{2}}{2 !}\right) e^{r}$,

$k_{3}=\left(1-t+\frac{t^{2}}{2 !}-\frac{t^{3}}{3 !}\right) e^{r}$,

The closed form solution will be

$k(r, t)=e^{r-t}$.

Example 3. Consider the following homogeneous fifth order KDV equation

$k_{t}=-k_{r}-k^{2} k_{r r}-k_{r} k_{r r}+20 k^{2} k_{r r r}-k_{r r r r}$,

With initial condition $k(r, 0)=\frac{1}{r}$

The initial problem is 
$L\left(k_{0}(r, t)\right)=0$,

Then, we get

$k_{0}(r, t)=\frac{1}{r}$

And the first iteration will be:

$k_{1_{t}}=-k_{0_{r}}-k_{0}{ }^{2} k_{0_{r r}}-k_{0_{r}} k_{0_{r r}}+20{k_{0}}^{2} k_{0_{r r r}}-k_{0_{r r r r}}$,

(37)

Applying ET to both aspects

$\varepsilon\left[k_{1 t}\right]=\varepsilon\left[-k_{0_{r}}\right]-\varepsilon\left[k_{0}^{2} k_{0 r r}\right]-\varepsilon\left[k_{0_{r}} k_{0 r r}\right]+$ $\varepsilon\left[20 k_{0}^{2} k_{0 r r r}\right]-\varepsilon\left[k_{0_{r r r r r}}\right]$,

(38)

Then we have:

$k_{1}=\frac{1}{r}\left(1+\frac{t}{r}\right)$,

$k_{2}=\frac{1}{r}\left(1+\frac{t}{r}+\frac{t^{2}}{r^{2}}-\frac{2 t^{3}}{3 r^{6}}-\frac{3 t^{4}}{2 r^{7}}-\frac{360 t^{3}}{r^{7}}-\frac{120 t^{4}}{r^{8}}\right)$,

$k_{3}=\left(1+\frac{t}{r}+\frac{t^{2}}{r^{2}}+\frac{t^{3}}{r^{4}}-\frac{13 t^{4}}{6 x^{8}}-\frac{26 t^{5}}{5 x^{9}}-\frac{5 t^{4}}{3 x^{11}}-\frac{480 t^{6}}{x^{11}}-\frac{12 t^{7}}{7 x^{11}}-\right.$ $\frac{1152 t^{5}}{x^{10}}-\frac{5 t^{6}}{x^{10}}-\frac{1740 t^{4}}{x^{9}}-\frac{7236000 t^{4}}{x^{13}}-\frac{23912 t^{5}}{x^{13}}-\frac{116 t^{6}}{9 x^{13}}-$ $\frac{8600 t^{4}}{x^{12}}-\frac{106 t^{5}}{15 x^{12}}-\frac{1200 t^{7}}{7 x^{12}}+\frac{1400400 t^{8}}{x^{17}}+\frac{4320 t^{9}}{x^{17}}+\frac{19137600 t^{7}}{7 x^{16}}+$ $\frac{16 t^{9}}{x^{16}}+\frac{3624000 t^{6}}{x^{15}}+\frac{20360 t^{7}}{x^{15}}+\frac{485 t^{8}}{12 x^{15}}-\frac{1065600 t^{5}}{x^{14}}+\frac{7140 t^{6}}{x^{14}}+$ $\frac{44 t^{7}}{21 x^{14}} \frac{3748032000 t^{7}}{7 x^{20}}-\frac{2815800 t^{8}}{x^{20}}-\frac{572410 t^{9}}{27 x^{20}}-\frac{247 t^{10}}{4 x^{20}}+$ $\frac{43920000 t^{7}}{7 x^{19}}+\frac{153280 t^{8}}{3 x^{19}}+\frac{11599 t^{9}}{162 x^{19}}+\frac{471200 t^{7}}{21 x^{18}}+\frac{1439 t^{8}}{18 x^{18}}+$ $\frac{296000 t^{9}}{x^{18}}+\frac{1112 t^{7}}{63 x^{17}}+-\frac{1762559632 t^{11}}{33 x^{24}}-\frac{49082111776 t^{10}}{135 x^{23}}-$ $\frac{15508800 t^{11}}{11 x^{23}}-\frac{828480000 t^{9}}{x^{22}}-\frac{6756960 t^{10}}{x^{22}}-\frac{130140 t^{11}}{11 x^{22}}-$ $\frac{844992000 t^{8}}{x^{21}}-\frac{28950400 t^{9}}{3 x^{21}}-\frac{37148 t^{10}}{x^{21}}-\frac{351 t^{11}}{11 x^{21}} \frac{67184640000 t^{10}}{x^{27}}+$ $\frac{14587776000 t^{11}}{11 x^{27}}+\frac{6517600 t^{12}}{x^{27}}+\frac{111780 t^{13}}{13 x^{27}}+\frac{671846400 t^{10}}{x^{26}}+$ $\frac{84672000 t^{11}}{11 x^{26}}+\frac{23800 t^{12}}{x^{26}}+\frac{243 t^{13}}{13 x^{26}}+\frac{2280960 t^{10}}{x^{25}}+\frac{5^{26} 3440 t^{11}}{33 x^{25}}+$ $\frac{25 t^{12}}{x^{25}}+\frac{9728 t^{10}}{3 x^{24}} \frac{34214400000 t^{13}}{13 x^{30}}+\frac{23328000000 t^{12}}{x^{29}}+$ $\frac{1321920000 t^{13}}{13 x^{29}}+\frac{755827200000 t^{11}}{11 x^{28}}+\frac{673488000 t^{12}}{x^{28}}+$ $\left.\frac{18565200 t^{13}}{13 x^{28}}\right) \frac{1}{r}$

We can find another iteration but the third iteration is too long, so, we stop then the closed form solution will be $k(r, t)=\frac{1}{x-t}$.

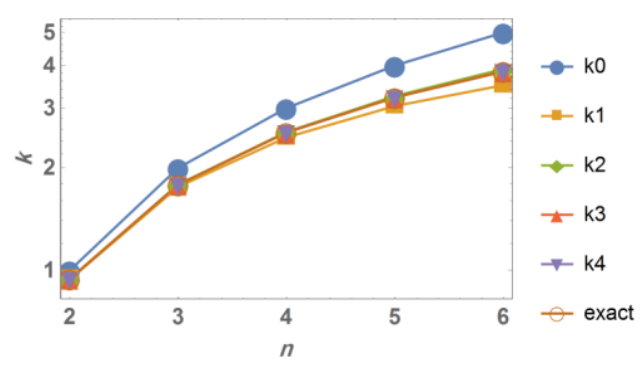

Figure 1. Comparison of the approximate solution $k_{0}, k_{1}, k_{2}, k_{3}$ with the exact solution(example 1)

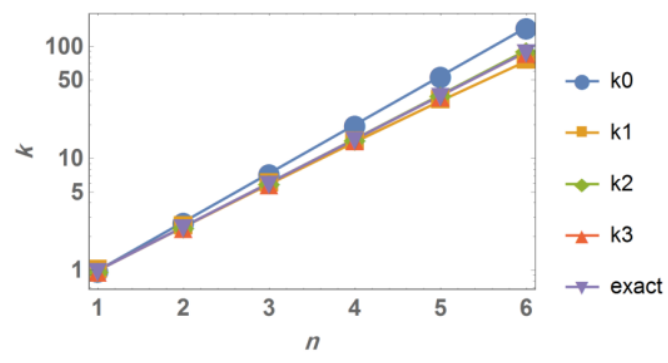

Figure 2. Comparison of the approximate solution $k_{0}, k_{1}, k_{2}, k_{3}$ with the exact solution (example 2)

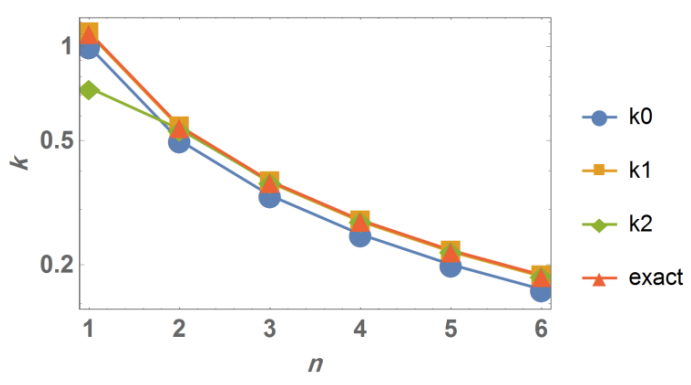

Figure 3. Comparison of the approximate solution $k_{0}, k_{1}, k_{2}, k_{3}$ with the exact solution (example 3 )

In the Figures 1, 2, 3 we compared some of the iterations with the exact solution so that we find that as we progress with the iterations, we will approach the exact solution.

\section{Conclusion}

In this research, a new method has been applied, which is to combine the TAM with the Elzaki transformation to $\mathrm{KdV}$ equations, and we have concluded that the method used is an effective method for finding the solution. Moreover, the problems examined show that the method used is a very powerful integrated transformation method for solving the $\mathrm{KdV}$ equations. Using this method makes us know how powerful this method is because all the problems discussed lead to accurate solutions with a small number of iterations.

\section{REFERENCES}

[1] AL-Jawary.M.A.( 2018). Radhi. Gh. H.; Ravnik. J. DaftardarJafari method for solving nonlinear thin film flow problem. Arab Journal of Basic and Applied Sciences. 25, 1, 20-27.

[2] AL-Jawary. M.A.; Mohammed.A.S. (2017). A Semi-Analytical Iterative Method for Solving Linear and Nonlinear Partial Differential Equations. International Journal of Science and Research. 6, 5,978982.

[3] Tawfiq. L. N.; Hasan. M. A. (2019). Evaluate the Rate of Pollution in Soil using Simulink Environment. Ibn Al Haitham Journal for Pure and Applied Science. 32, 1, 132-138.

[4] Adnan.F.A.; Abdul Hadi A. M. (2019). Peristaltic Flow of the Bingham Plastic Fluid in a Curved Channel. Ibn Al Haitham Journal for Pure and Applied Science. 32, 3, 140-152

[5] Kaya.D.; Aassila. M. (2002). An application for a generalized KdV equation by the decomposition method. Physics Letters A. 299, 2-3, 201-206.

[6] Mirgolbabaei. H.; Ganji. D.D. (2009). Application of Homotopy Perturbation Method to Solve Combined Korteweg de Vries-Modified Korteweg de Vries Equation. Journal of Applied Sciences. 9, 19, 3587-3592.

[7] Yassein. S. M.; Aswhad. A. A. (2019). Efficient Iterative Method for Solving Korteweg-de Vries Equations. Iraqi Journal of Science. $60,7,1575-1583$.

[8] Hendi. F.A. (2011). Laplace Adomian Decomposition Method for Solving the Nonlinear Volterra Integral Equation with Weakly 
Kernels. Studies in Nonlinear Sciences. 2, 4, 129-134.

[9] Ahmed.Sh.Sh.; Salih. Sh. A. H.; Ahmed. M. R. (2019). Laplace Adomian and Laplace Modified Adomian Decomposition Methods for Solving Nonlinear IntegroFractional Differential Equations of the Volterra-Hammerstein Type. Iraqi Journal of Science. 60, 10, 2207-2222.

[10] Eljaily. M. H.; Elzaki. T. M. (2015). Homotopy Perturbation Transform Method for Solving (KDV) Equation, Pure and Applied Mathematics Journal. 4, 6, 264-268.

[11] Suleman. M.; Wu. Q.; Abbas. Gh. (2016). Approximate analytic solution of $(2+1)$ dimensional coupled differential Burger's equation using Elzaki Homotopy Perturbation Method. Alexandria Engineering Journal. 55, 2, 1817-1826.

[12] Nuruddeen. R. I.; Nass. A. M. (2016). Aboodh Decomposition Method and its Application in Solving Linear and Nonlinear Heat Equations. European Journal of Advances in Engineering and Technology. 3, 7, 34-37.

[13] Elzaki. T. M.; Chamekh. M. (2018). Solving Nonlinear Fractional Differential Equations using a New Decomposition Method. Universal Journal of Applied Mathematics \& Computation. 6, 27-35

[14] Elijah. I. O. Approximate Analytical Solution of Nonlinear Differential Equations using Elzaki Transform. Case study: Korteweg-de Vries (KdV) Equations. Master's Thesis, Lappeenranta University of Technology, (2018).

[15] Temimi. H.; Ansari. A. R. (2011). A Semi Analytical Iterative Technique for Solving Nonlinear Problems. Computers and Mathematics with Applications. 61, 2, 203- 210.

[16] Elzaki. T. M. (2011). The New Integral Transform "ELzaki Transform". Global Journal of Pure and Applied Mathematics. $7,1,57-64$. 